\title{
Validade e Concordância do registro em prontuário do uso de serviços da Rede de Atenção à Saúde por idosos
}

\author{
Validity and consistency of the entries in the Health Care Network's \\ patient records for the use of services by the elderly
}

Vanessa Carvalho Leite Gama Rocha (https://orcid.org/0000-0002-3820-3597) ${ }^{1}$ Daniele Sirineu Pereira (https://orcid.org/0000-0002-4868-9244) ${ }^{1}$

Maria Jaqueline Pereira (https://orcid.org/0000-0002-5779-2673) ${ }^{1}$

Silvia Lanziotti Azevedo da Silva (https://orcid.org/0000-0002-2323-2029) ${ }^{1}$
${ }^{1}$ Instituto de Ciências da Motricidade, Curso de Fisioterapia, Universidade Federal de Alfenas. Av. Jovino Fernandes Sales, Santa Clara. 31270-901 Alfenas MG Brasil. nessaclgr@hotmail.com
Abstract The scope of this article is to verify the validity and consistency of entries in medical records and self-reporting about the use of the health services by elderly users of the Health Care Network. It involved a cross-sectional, population-based observational study. Entries in medical, dental, home visit, referral to the secondary sector and hospitalization appointment records for the years 2015 and 2016 were evaluated. The concordance percentage, Kappa coefficient, sensitivity, specificity and predictive values for each item were also analyzed. The highest concordance percentage (81.93\%) with a significant Kappa coefficient ( $p=0.03$ ) was for hospitalization. The dentistry appointments revealed a higher Kappa coefficient $(k=0.271)$ with significance of $p=0.01$. The analyses showed high specificity in the hospitalization records (83\%), and a greater sensitivity for home visits (74\%). Positive predictive values were low for hospitalization (8\%), and negative predictive values were low for medical appointments (17\%). The low concordance between the use of the self-reported health service and entries in medical records highlight one of the possible causes of the fragmentation in continuity of care. Key words Primary health care, Public health, Health services, Validity of tests, Self-reporting
Resumo O objetivo deste artigo é verificar a validade e a concordância entre registros em prontuários e autorrelato do uso dos serviços de saúde por idosos usuários da Rede de Atenção à Saúde. Estudo observacional transversal de base populacional. Foram avaliados registros em prontuário e autorrelato de consultas médicas, odontológicas, visitas domiciliares, encaminhamentos para consultas no setor secundário e hospitalizações nos anos de 2015 e 2016. Foram analisados percentuais de concordância, estatística Kappa, sensibilidade, especificidade e valores preditivos para cada um dos itens. O maior percentual de concordância $(81,93 \%)$ com Kappa significativo $(p=0,03)$ foi para o item "hospitalização". As consultas odontológicas apresentaram maior Kappa $(k=0,271)$, significativo $(p=0,01)$. As análises demonstraram alta especificidade nos registros de hospitalização (83\%), e maior sensibilidade para visitas domiciliares (74\%). Os valores preditivos positivos foram baixos para hospitalização (8\%), e os negativos baixos para consultas médicas (17\%), A baixa concordância entre o uso do serviço de saúde autorrelatado e registros em prontuário apontam para uma das possiveis causas da fragmentação da continuidade do cuidado.

Palavras-chave Atenção Primária à Saúde, Saúde pública, Serviços de saúde, Validade dos testes, Autorrelato 


\section{Introdução}

O envelhecimento populacional é uma realidade, e o Brasil registra uma das maiores taxas deste fenômeno da América Latina. Espera-se que, em 2050, pelo menos $20 \%$ da população total seja composta por idosos ${ }^{1,2}$. Tal fenômeno gera demandas sociais, em escala mundial, que requerem políticas adequadas, principalmente em relação aos serviços de saúde, uma vez que a população idosa apresenta necessidades próprias e linhas de cuidado específicas ${ }^{3-5}$.

A busca por atendimentos de saúde por parte dos idosos é expressiva em todos os níveis da Rede de Atenção à Saúde (RAS) ${ }^{6}$. Em muitos casos, esta população utiliza os níveis Secundário e Terciário, de maior complexidade, como porta de entrada para o Sistema, mas grande parte de suas demandas poderiam ser resolvidas na Atenção Primária à Saúde (APS) ${ }^{7}$. Tal busca pode ocorrer devido ao desconhecimento dos serviços oferecidos no primeiro nível da RAS e pela persistência de um pensamento hospitalocêntrico, assistencialista e altamente especializado dos idosos ${ }^{8}$.

A educação desta população acerca da importância do cuidado preventivo, informações sobre serviços oferecidos em cada nível e a oferta de cuidado de qualidade na APS são importantes para que a sua resolutividade desejada seja alcançada ${ }^{9}$, uma vez que o papel deste primeiro nível é coordenar o cuidado e ser capaz de resolver até $85 \%$ das demandas ${ }^{10}$.

É importante o monitoramento das condições de uso dos serviços de saúde da população, e uma das formas mais tradicionais para tal são os inquéritos populacionais de saúde, usados de forma crescente para avaliação do ponto de vista do usuário ${ }^{11}$. O autorrelato é uma ferramenta capaz de oferecer suporte para a reorganização dos serviços, por meio de informações pertinentes e confiáveis, sendo amplamente utilizada em estudos nacionais ${ }^{12-14}$ e internacionais ${ }^{15,16}$.

Outra forma de avaliação do serviço de saúde é a análise de dados pertencentes à prontuários. Trata-se de um documento único, constituído por um conjunto de informações registradas, acontecimentos e situações sobre a saúde do usuário, assim como a assistência prestada a ele, possuindo caráter legal, sigiloso e científico ${ }^{17}$. Uma das grandes dificuldades encontradas nas pesquisas que envolvem análise do prontuário está na qualidade das informações, muitas vezes incompletas ou ilegíveis ${ }^{18-20}$. Os dados registrados em prontuários na APS mostraram que as ações têm sido feitas de forma fragmentada, indican- do a falta de integração e articulação entre profissionais na construção do Projeto Terapêutico Singular ${ }^{21}$.

A comparação dos dados de prontuários na APS com o autorrelato do uso do serviço pelos usuários idosos é válida por possibilitar a avaliação da qualidade e segurança dos registros, e a verificação da coerência entre o que está inserido no prontuário com o que é relatado pelo usuário ${ }^{22}$.

O objetivo do estudo foi comparar as informações pertencentes à prontuários de Unidades Básicas de Saúde da Família (UBSF) com o autorrelato do uso dos serviços públicos de saúde pelos idosos adscritos à Estratégia Saúde da Família (ESF).

\section{Materiais e métodos}

\section{Desenho e local de realização do estudo}

Trata-se de um estudo observacional transversal com caráter metodológico de base populacional dos idosos do município de Alfenas, localizado no sul do Estado de Minas Gerais. O município, segundo dados do Instituto Brasileiro de Geografia e Estatística (IBGE) de 2010, apresenta população total de 73.774 habitantes, sendo $8.421(11,41 \%)$ acima de 60 anos. A cobertura da ESF no município de Alfenas é de aproximadamente $70 \%$ da população, e, no momento do recrutamento da amostra, contava com 4.005 (47,55\%) idosos cadastrados nas 14 Equipes da ESF local, divididas entre 12 Unidades Básicas de Saúde da Família (UBSF). As 12 UBSF são subdividas em 4 regiões de saúde, com 3 em cada, contendo em cada região uma unidade que concentra maior número de idosos. O município foi selecionado para a realização do estudo por possuir uma parcela de idosos semelhante aos valores nacionais, com quase 50\% dela adscrita à ESF, condição fundamental para o andamento do estudo.

\section{Processo de seleção e caracterização da amostra}

Baseado em cálculo amostral para amostras aleatórias estratificadas por território e regiões de saúde, com 95\% de confiança e 5\% de erro, a amostra para todas as UBSF foi composta por 350 idosos. Para o presente estudo, foram selecionadas 6 UBSF, uma em cada quadrante do município, sendo 2 contemplados com 2 unidades, por abrangerem território menor, garantindo as- 
sim a representatividade. Pelo cálculo, a amostra para estas 6 UBSF seria composta por 193 idosos. Para garantia de correspondência entre o registro no prontuário e a possibilidade de entrevista do idoso, e também considerar possíveis perdas, a amostra final do presente estudo foi composta por 211 idosos.

Em todas as Unidades, os idosos que responderam ao questionário sobre o uso dos serviços de saúde e cujos prontuários foram acessados foram sorteados aos poucos, a partir de uma lista composta por todos os aqueles adscritos à cada equipe incluída no estudo, até que fosse atingido o número determinado pelo cálculo amostral.

Foram incluídos na amostra idosos que concordaram em receber a visita dos entrevistadores em seu domicílio, e cujos prontuários estavam disponíveis na unidade. Foram excluídos idosos com alteração cognitiva detectada pelo Mini Exame do Estado Mental (MEEM), segundo pontos de corte de Bertolucci et al. ${ }^{23}$, acamados ou cadeirantes, com sequelas de Acidente Vascular Encefálico (AVE) grave ou doença de Parkinson. As entrevistas foram realizadas com o próprio idoso, sem interferência do cuidador.

Para a caracterização da amostra foram consideradas variáveis sociodemográficas, sexo, idade, nível de escolaridade, raça, estado civil, renda, posse de casa própria e número de moradores da residência.

\section{Análise de prontuário}

A análise de prontuário foi feita seguindo roteiro estruturado, contendo número de consultas médicas com o médico da UBSF, consultas odontológicas com o dentista da UBSF, visitas domiciliares realizadas pelos profissionais da ESF, encaminhamentos para consultas no nível secundário da Rede de Atenção (RAS) e hospitalizações. Foram considerados os registros dos anos de 2015 e 2016.

Todas as análises dos documentos foram feitas por dois pesquisadores treinados, com presença de profissionais responsáveis pelo serviço.

\section{Autorrelato do uso do serviço de saúde}

Para avaliação do uso do serviço de saúde relatado pelos idosos, foi aplicado um questionário semiestruturado, entre 2015 e 2016, em horário não agendado e por pesquisadores treinados. Durante a entrevista, o idoso foi questionado quanto à realização de consultas médicas e odon- tológicas na UBSF, recebimento de visita domiciliar por algum membro da ESF, encaminhamento para consultas no nível secundário da RAS, e hospitalizações. Foi considerada hospitalização quando a permanência em ambiente hospitalar fosse superior a 24 horas.

\section{Comparação entre as informações para avaliação da validade e concordância entre os métodos}

Foram comparadas as informações registradas em prontuário e autorrelatadas por idosos acerca da realização de consultas médicas, consultas odontológicas, visitas domiciliares, encaminhamento para consultas no nível secundário e hospitalização.

Por ser o método mais utilizado em pesquisas com idosos sobre esta temática, o autorrelato foi considerado padrão-ouro ou medida real do uso dos serviços de saúde, sendo o registro no prontuário da Unidade comparado a ele para avaliação de sua validade e concordância.

\section{Análise estatística}

A descrição da amostra foi feita por medidas de média e desvio padrão para as variáveis contínuas e percentuais para as variáveis categóricas. Para avaliação da validade e concordância entre os registros em prontuário e autorrelato dos idosos foram considerados consultas médicas, consultas odontológicas, visitas domiciliares, encaminhamentos para consultas no setor secundário e hospitalização. As variáveis relacionadas ao uso dos serviços de saúde foram categorizadas dummy, com opções "sim" ou "não" como resposta.

Para avaliação da concordância foi aplicada estatística Kappa e o percentual de concordância entre as duas formas de avaliação de uso dos serviços de saúde. Para análise dos valores de Kappa, valores $<0$ foram considerados "nenhuma concordância", entre 0-0,19 "pobre", entre 0,2-0,39 "ligeira", entre 0,40-0,59 "moderada", entre 0,600,79 "substancial" e entre $0,80-1,0$ perfeita concordância $^{24}$.

Para avaliação da validade do registro em prontuário comparado ao autorrelato foram realizadas análises da sensibilidade, especificidade, valor preditivo positivo e valor preditivo negativo.

As análises foram realizadas utilizando o software SPSS versão 18.0, e foi considerado nível de siginificância $\alpha=0,05$ e Intervalo de Confiança de 95\% (IC95\%). 


\section{Considerações éticas}

O presente estudo foi aprovado pelo Comitê de Ética em Pesquisa da Universidade Federal de Alfenas. Os idosos participantes da pesquisa assinaram Termo de Consentimento Livre e Esclarecido (TCLE). Para o acesso aos prontuários, os responsáveis pelas UBSF assinaram o Termo de Consentimento de Utilização de Dados (TCUD).

\section{Resultados}

A amostra foi composta por $63,5 \%$ idosos do sexo feminino, com idade média 70,24 $( \pm 6,48)$ anos. Demais características sociodemográficas e clínicas da amostra estão inseridas na Tabela 1.

Em relação ao relato do uso dos serviços de saúde pelas duas formas de informação deste avaliadas no estudo, os percentuais de "sim" ou "não" para cada uma estão registrados na Tabela 2. Os maiores valores de Percentual de Concordância observados foram para consultas médicas $(85,7 \%)$ e hospitalização $(81,9 \%)$. O menor Percentual registrado foi para visitas domiciliares $(28,9 \%)$, onde os idosos relatam mais visitas que o registrado no prontuário (Tabela 2).

$O$ índice de Kappa foi utilizado a fim de apontar o quanto o Percentual de Concordância pode ser devido ao acaso, assim como a magnitude da concordância entre os dois métodos. A concordância entre registros e relatos para consultas odontológicas apresentou maior magnitude do Kappa, mas ainda considerada ligeira $(\mathrm{k}=$ $0,27)$, embora significativa $(\mathrm{p}=0,01)$. O valor do Kappa para hospitalização $(\mathrm{k}=0,10)$ foi considerado pobre e significativo $(\mathrm{p}=0,03)$. Não foram significativos os valores do Kappa para consultas médicas, encaminhamentos para o nível secundário e visita domiciliar (Tabela 3).

A Tabela 4 apresenta análise de validade baseada nos valores de sensibilidade, especificidade e valores preditivos positivos e negativos do registro em prontuário comparado ao autorrelato (padrão-ouro). Em relação à hospitalização, a especificidade foi alta $(83 \%)$, indicando que quando há ausência de registro no prontuário deste evento, não significa que ele realmente não ocorreu (falso-negativo). As consultas odontológicas apresentam maior equilíbrio entre sensibilidade e especificidade, indicando a melhor qualidade do registro em prontuário, entre todos os observados. Já os encaminhamentos para consultas no nível secundário apresentam sensibilidade mais alta (74\%) indicando que o registro em prontu-
Tabela 1. Caracterização sociodemográfica e clínica da amostra de idosos adscritos à Estratégia de Saúde da Família $(\mathrm{n}=211)$.

\begin{tabular}{|c|c|c|}
\hline Variável & $\%(\mathbf{n})$ & $\begin{array}{c}\text { média } \pm \\
\text { desvio- } \\
\text { padrão }\end{array}$ \\
\hline \multicolumn{3}{|l|}{ Sexo: } \\
\hline Masculino & $36,5(77)$ & \\
\hline Feminino & $63,5(134)$ & \\
\hline Idade & & $70,2 \pm 6,4$ \\
\hline \multicolumn{3}{|l|}{ Estado Civil: } \\
\hline Casado & $64,5(136)$ & \\
\hline Solteiro & $4,3(9)$ & \\
\hline Divorciado & $5,7(12)$ & \\
\hline Viúvo & $25,5(54)$ & \\
\hline \multicolumn{3}{|l|}{ Raça: } \\
\hline Branca & $62,1(131)$ & \\
\hline Negra & $9,5(20)$ & \\
\hline Mulata & $28,0(59)$ & \\
\hline Indígena & $0,4(1)$ & \\
\hline \multicolumn{3}{|l|}{ Escolaridade: } \\
\hline Nunca foi à escola & $26,5(56)$ & \\
\hline $\begin{array}{l}\text { Curso de Alfabetização } \\
\text { para adultos }\end{array}$ & $1,9(4)$ & \\
\hline Primário & $58,8(124)$ & \\
\hline Ginásio & $9,5(20)$ & \\
\hline Científico & $2,4(5)$ & \\
\hline Superior & $0,9(2)$ & \\
\hline \multicolumn{3}{|l|}{ Realiza Atividade } \\
\hline \multicolumn{3}{|l|}{ Remunerada: } \\
\hline Não & $85,3(180)$ & \\
\hline Sim & $14,7(31)$ & \\
\hline $\begin{array}{l}\text { Número de Moradores } \\
\text { da Residência: }\end{array}$ & & $2,7 \pm 1,4$ \\
\hline \multicolumn{3}{|l|}{ Casa Própria: } \\
\hline Não & $9,0(19)$ & \\
\hline $\operatorname{Sim}$ & $9,0(192)$ & \\
\hline \multicolumn{3}{|l|}{$\begin{array}{l}\text { Dinheiro Suficiente para } \\
\text { as Despesas: }\end{array}$} \\
\hline Não & $46,6(98)$ & \\
\hline Sim & $53,4(113)$ & \\
\hline $\begin{array}{l}\text { Número de Doenças } \\
\text { Crônicas: }\end{array}$ & & $3,9 \pm 2,5$ \\
\hline
\end{tabular}

ário pode ser medida de rastreio para o uso de outros níveis da rede de atenção, embora muitos registros possam não representar realmente $o$ relato destes encaminhamentos para consultas em outros níveis pelos idosos (falsos-positivos). A sensibilidade foi alta para as consultas médicas (92\%), indicando também que muitos registros 
Tabela 2. Descrição do uso de serviços de saúde registrados em prontuário e autorrelato pelos idosos $(\mathrm{n}=211)$.

\begin{tabular}{lccc}
\hline Uso dos Serviços de Saúde & $\begin{array}{c}\text { Registro no } \\
\text { Prontuário }(\% / \mathbf{n})^{*}\end{array}$ & $\begin{array}{c}\text { Auto-Relato dos } \\
\text { Idosos }(\% / \mathbf{n})^{* *}\end{array}$ & $\begin{array}{c}\text { Percentual de } \\
\text { Concordância } \\
\text { Observada }(\%)\end{array}$ \\
\hline Consultas Médicas: & $9(19)$ & $8,1(17)$ & 85,78 \\
$\quad$ Não & $91(192)$ & $91,9(194)$ & \\
Sim & & & 75,2 \\
Consultas Odontológicas: & $89,1(188)$ & $71,6(151)$ & \\
$\quad$ Não & $10,9(23)$ & $28,4(60)$ & 28,9 \\
Sim & $76,3(161)$ & $8,1(17)$ & \\
Visitas Domiciliares: & $23,2(50)$ & $91,9(194)$ & 57,8 \\
$\quad$ Não & & & \\
Sim & $44,5(94)$ & $30,8(65)$ & 81,9 \\
Consultas no Setor Secundário: & $55,5(117)$ & $69,2(146)$ & \\
$\quad$ Não & & & \\
Sim & $97,2(205)$ & $82,9(175)$ & $17,1(36)$ \\
Hospitalização: & $2,8(6)$ & & \\
$\quad$ Não &
\end{tabular}

Tabela 3. Índice de Concordância de Kappa entre autorrelato por usuários idosos e registro em prontuários na APS do uso dos serviços de saúde $(\mathrm{n}=211)$.

\begin{tabular}{|c|c|c|c|c|c|c|c|}
\hline \multicolumn{8}{|c|}{ Prontuário } \\
\hline Autorrelato & Não & Sim & Total & Kappa & $\begin{array}{c}\text { Magnitude } \\
\text { Kappa }\end{array}$ & $\begin{array}{l}\text { p valor } \\
\text { Kappa }\end{array}$ & $\begin{array}{l}\text { IC95\% } \\
\text { Kappa }\end{array}$ \\
\hline \multicolumn{8}{|l|}{ Consulta médica } \\
\hline Não & 3 & 14 & 17 & & & & \\
\hline Sim & 16 & 178 & 194 & 0,09 & Pobre & 0,19 & $0,04-0,22$ \\
\hline Total & 19 & 192 & 211 & & & & \\
\hline \multicolumn{8}{|l|}{ Consulta Odontológica } \\
\hline Não & 144 & 7 & 151 & & & & \\
\hline Sim & 44 & 16 & 60 & 0,27 & Ligeira & $0,001^{*}$ & $0,15-0,38$ \\
\hline Total & 188 & 23 & 211 & & & & \\
\hline \multicolumn{8}{|l|}{ Visita Domiciliar } \\
\hline Não & 14 & 3 & 17 & & & & \\
\hline Sim & 147 & 47 & 194 & 0,01 & Pobre & 0,56 & $0,03-0,56$ \\
\hline Total & 161 & 50 & 211 & & & & \\
\hline \multicolumn{8}{|c|}{ Encaminhamento Setor Secundário } \\
\hline Não & 35 & 30 & 65 & & & & \\
\hline Sim & 59 & 87 & 146 & 0,12 & Pobre & 0,07 & $0,01-0,24$ \\
\hline Total & 94 & 117 & 211 & & & & \\
\hline \multicolumn{8}{|l|}{ Hospitalização } \\
\hline Não & 172 & 3 & 175 & & & & \\
\hline Sim & 33 & 3 & 36 & 0,10 & Pobre & $0,03^{*}$ & $0,01-0,18$ \\
\hline Total & 205 & 6 & 211 & & & & \\
\hline
\end{tabular}

podem ser falsos-positivos. A especificidade foi baixa para visitas domiciliares $(8 \%)$, indicando pouca precisão em relação a este serviço.
Em relação aos valores preditivos, a hospitalização apresenta valor preditivo negativo alto (98\%), indicando que, quando não há registro, 
Tabela 4. Validade do registro em prontuário em relação ao autorrelato do uso dos serviços de saúde por idosos adscritos à Estratégia de Saúde de Família: sensibilidade, especificidade, valor preditivo positivo e valor preditivo negativo.

\begin{tabular}{lcccc}
\hline \multirow{2}{*}{ Uso do Serviços } & Sensibilidade & Especificidade & $\begin{array}{c}\text { Valor Preditivo } \\
\text { Positivo }\end{array}$ & $\begin{array}{c}\text { Valor Preditivo } \\
\text { Negativo }\end{array}$ \\
\cline { 2 - 5 } & $0,92(92 \%)$ & $0,15(15 \%)$ & $0,91(91 \%)$ & $0,17(17 \%)$ \\
Consultas Médicas & $0,69(69 \%)$ & $0,60(60 \%)$ & $0,26(26 \%)$ & $0,75(75 \%)$ \\
Consultas Odontológicas & $0,94(94 \%)$ & $0,08(8 \%)$ & $0,24(24 \%)$ & $0,82(82 \%)$ \\
Visita Domiciliar & $0,74(74 \%)$ & $0,37(37 \%)$ & $0,59(59 \%)$ & $0,37(37 \%)$ \\
Serviço Secundário & $0,50(50 \%)$ & $0,83(83 \%)$ & $0,08(8 \%)$ & $0,98(98 \%)$ \\
Hospitalização & & & & \\
\hline
\end{tabular}

é provável que o idoso não relate este evento. Os encaminhamentos para consultas no nível secundário têm valor preditivo negativo de $37 \%$, indicando que o idoso muitas vezes recebe o encaminhamento, mas ele não é registrado (Tabela 4).

\section{Discussão}

Os resultados do presente estudo apontam para uma baixa concordância entre o autorrelato e os registros em prontuário do uso de serviços públicos de saúde pelos idosos, e a validade deste registro quando comparadas às informações coletadas diretamente com os mesmos. Em relação à amostra, com predomínio de mulheres, casadas e brancas, foi semelhante à de outros estudos que avaliaram o uso do serviço de saúde na APS por idosos $^{13,14}$.

Em relação à análise de concordância, o maior valor de Kappa foi observado para consultas odontológicas, considerado ligeiro pela classificação adotada, porém significativo. Este resultado pode ser explicado pelo fato do prontuário ser separado, o que facilita o controle de registro de atendimento pelo profissional dentista. Para os demais profissionais como médico, enfermeiro e técnico em enfermagem, que compõem a ESF, o prontuário é único e na maioria dos locais ainda não é informatizado, o que predispõe a subnotificação.

Um estudo realizado em cinco ESF de municípios do Rio Grande do Sul avaliou registros em prontuários baseado nos seguintes atributos: identificação completa do indivíduo; dados referentes ao prestador de serviço; informações mínimas para identificação do atendimento; realização de consulta médica, enfermagem, e outros profissionais; legibilidade; presença de lista de problemas; relato de alergias a medicamentos; e recebimento de visita domiciliar. A falta de padrão das informações e registros incompletos dificultaram o acesso às informações do usuário, comprometidas de forma geral ${ }^{21}$. Uma possível alternativa para minimizar o problema seria a implementação do prontuário eletrônico, que faz com que este deixe de ser um documento passivo, difícil de entender, afastado do usuário, e se torne ativo, centralizando serviços e informações confiáveis $^{20,25,26}$.

A alta sensibilidade observada nos registros de consultas médicas e visitas domiciliares, indica muitos "falsos positivos", ou seja, os registros podem não corresponder ao relatado pelo idoso. O coeficiente de Kappa não significativo e considerado "pobre" para estes pontos reforça o resultado, mostrando que não há correspondência entre os dados. Este resultado chama a atenção novamente para a falta de qualidade do registro, uma vez que o prontuário é um importante instrumento no cuidado e acompanhamento do paciente, como demonstrado em revisão da literatura acerca da relevância deste documento nas leis vigentes, concluindo que o mesmo representa uma ferramenta simples, útil, e, quando preenchida de maneira correta, pode ser capaz de refletir uma imagem precisa acerca da situação de saúde dos usuários ${ }^{27}$. Em uma avaliação de prontuários de uma instituição pública, os principais problemas encontrados foram a ilegibilidade das informações e o preenchimento incorreto ou incompleto $^{20}$.

Em relação ao uso de outros níveis da RAS, observou-se uma baixa concordância entre hospitalização, com Kappa classificado como pobre, porém significativo; e encaminhamentos para consultas no nível secundário, este último com Kappa não significativo. Este resultado pode ser 
remetido a uma falha no sistema e referência e contrarreferência e a procura direta por atendimento em níveis de maior complexidade. A percepção dos profissionais de saúde acerca da referência e contrarreferência em um município de Minas Gerais revelou que 59,1\% dos usuários não retornam ao atendimento na APS com contrarreferência e $98,9 \%$ dos profissionais deste nível relataram encaminhar os usuários para níveis mais complexos ${ }^{28}$.

A possível falta de resolutividade das ações da APS pode estar na deficiência da "alfabetização em saúde". Trata-se da capacidade de obter, processar e compreender informações e serviços básicos necessários para gerenciar a saúde e tomar decisõe ${ }^{29}$. Para avaliar a satisfação de usuários de um seguro saúde e seu nível de "alfabetização em saúde", foram enviados inquéritos a 31.000 usuários de 10 diferentes estados nos Estados Unidos e, ao final, concluiu-se que com intervenções educacionais eficazes e investimento em serviços preventivos, seria possível alcançar melhor satisfação e atendimento dos usuários, reduzindo assim os gastos ${ }^{29}$.

As análises de especificidade, sensibilidade e valores preditivos feitas no presente estudo consideraram o autorrelato do uso dos serviços de saúde pelos idosos adscritos à ESF como padrão ouro. A Pesquisa Nacional de Saúde (PNS) é, atualmente, a principal fonte de informações sobre a autoavaliação e o uso dos serviços de saúde no Brasil, e também utiliza o autorrelato, o que justifica a escolha no presente estudo ${ }^{30}$.

A análise dos dados dos prontuários relacionados à hospitalizações mostrou uma especificidade alta e um baixo valor preditivo positivo, indicando que baseado na presença de seu registro em prontuário, não é possível saber se o idoso foi realmente hospitalizado. Tal achado pode ser remetido mais uma vez a falha na contrarreferência, quando o usuário procura outros níveis de atenção, recebe atendimento e não é contrarreferenciado à APS para continuidade do cuidado ${ }^{28}$.

$O$ registro dos encaminhamentos para consultas no nível secundário apresentou boa sensibilidade, podendo ser considerado medida de rastreio para o uso deste nível. Por outro lado, tal resultado pode apresentar "falsos positivos", indicando que o encaminhamento foi registrado, mas o idoso não utilizou o serviço, por isso pode não ter relatado a informação. A longa espera por atendimento na atenção secundária pode explicar a incoerência entre o número de encaminhamentos registrados e relatados ${ }^{31}$. Um sistema de vigilância e auditoria adotado na Costa Rica mostrou que o menor tempo de espera para atendimentos em outros níveis de atenção facilita a adesão do usuário ao tratamento, gerando benefícios para sua saúde ${ }^{32}$. No Brasil, iniquidades estão presentes em relação a realização das consultas médicas, sendo as possibilidades menores para aqueles com menor renda, o que representa importante barreira ao acesso a cuidados integrais pelo Sistema Único de Saúde (SUS), o que incluiria as consultas no nível secundário da RAS ${ }^{33}$.

As visitas domiciliares apresentaram grande número de falsos negativos. Na maioria dos casos, recebem cuidados domiciliares somente idosos restritos ao leito e com dificuldades importantes de locomoção $0^{34}$, grupo que foi excluído deste estudo. Por outro lado, os idosos provavelmente entenderam a questão da visita domiciliar como à ida ao domicilio por qualquer profissional na ESF, incluindo o Agente Comunitário de Saúde (ACS), profissional "ponte" entre usuários e ESF, e responsável também pelo cuidado ${ }^{35}$.

A evolução e o registro das visitas dos ACS são feitas em um formulário a parte, o que dificultou a contabilização das mesmas durante a análise de prontuário. Como compõe equipe mínima da ESF e participam do cuidado, o registro em conjunto poderia garantir a integralidade na assistência e facilitar a contabilização de atendimentos de toda a equipe, além de auxiliar na construção do Projeto Terapêutico Singular $(\mathrm{PTS})^{36}$.

Foram identificadas como limitações do estudo questões relacionadas aos prontuários existentes nas UBSF, a ilegibilidade dos registros e a baixa qualidade na conservação dos mesmos, que dificultaram a coleta das informações. São pontos fortes o fato deste ser um estudo com características inéditas, pois não foram encontrados, até o momento da realização deste trabalho, outros estudos que tivessem realizado comparação entre dados registrados em prontuários na APS e autorrelato do uso de serviços de saúde por idosos adscritos à ESF. Os resultados são capazes de incentivar a melhora da qualidade dos registros, sendo estes documentos capazes de ajudar a viabilizar o real atendimento integral e a coordenação do cuidado pela APS.

\section{Conclusão}

Podemos concluir que a falta de concordância e validade frágil entre o uso do serviço de saúde autorrelatado e os problemas relacionados aos registros em prontuário apontam uma das pos- 
síveis causas da quebra da continuidade do cuidado de pacientes na RAS, bem como a ausência da referência e contrarreferência. A padronização dos registros, que pode ser alcançada com auxílio da implantação do prontuário eletrônico, potencializaria o acompanhamento longitudinal, a coordenação do cuidado e a integralidade.

\section{Colaboradores}

Todos as autoras participaram de todas as etapas da produção do manuscrito e colaboraram com sua redação e aprovaram a versão final. As autoras SLA Silva e DS Pereira são coordenadoras das coletas e responsáveis pelo banco de dados; VCLG Rocha e MJ Pereira foram responsáveis pela coleta de dados, organização em planilhas, interpretação dos dados e resultados.

\section{Agradecimentos}

Os autores agradecem ao Conselho Nacional de Desenvolvimento Científico e Tecnológico e a Fundação de Amparo à Pesquisa do estado de Minas Gerais pelo apoio financeiro para o desenvolvimento da pesquisa. 


\section{Referências}

1. Küchemann BA. Envelhecimento populacional, cuidado e cidadania: velhos dilemas e novos desafios. Sociedade e Estado 2012; 27(1):165-180.

2. Carvalho JAM, Rodriguez-Wong L. A transição da estrutura etária da população brasileira na primeira metade do século XXI. Cad Saude Publica 2008; 24(3):597-605.

3. Pilger C, Menon MU, Mathias TAF. Utilização de serviços de saúde por idosos vivendo na comunidade. Rev. Esc. Enferm.USP 2013; 47(1):213-220.

4. Van der Wielen N, Channon AA, Falkingham J. Universal health coverage in the context of population ageing: What determines health insurance enrolment in rural Ghana? BMC Public Health 2018; 18(657):213.

5. Arensberg MB. Population aging: opportunity for business expansion, an invitational paper presented at the Asia-Pacific Economic Cooperation (APEC) International Workshop on Adaptation to Population Aging Issues, July 17, 2017, Ha Noi, Viet Nam. J Health Popul Nutr 2018; 10(37):1-12.

6. Brasil. Ministério da Saúde (MS). Política Nacional de Atenção Básica. Brasília: MS; 2012.

7. Silva RM, Andrade LOM. Coordenação dos cuidados em saúde no Brasil: o desafio federal de fortalecer a atenção primária à saúde. Physis, 2014; 24(4):12071228.

8. Onocko-Campos RT, Campos GWS, Ferrer AL, Corrêa CRS, Madureira PR, Gama CAP, Dantas DV, Nascimento R. Avaliação de estratégias inovadoras na organização da Atenção Primária à Saúde. Rev Saude Publica 2012; 46(1):43-50.

9. Zanon LR, Zanin L, Florio FM. Evaluation of the use of services provided by emergency care units in a Brazilian municipality. RGO, Rev Gaúch Odontol 2016; 64(4):411-414

10. Amorim DNP, Chiarello MD, Vianna LG, Moraes CF, Vilaça KHC. Interactions through conditions sensitive to primary attention of elderly persons in Brazil, 2003 to 2012. J Nurs UFPE on line 2017; 11(2):576583.

11. Szwarcwald CA, Malta DC, Pereira CA, Vieira MLFP, Conde WS, Souza Júnior PRB, Damacena GL, Azevedo LO, Silva GA, Theme Filha MM, Lopes CS, Romero DE, Almeida WS, Monteiro CA. Pesquisa Nacional de Saúde no Brasil: concepção e metodologia de aplicação. Cien Saude Colet 2014; 19(2):333-342.

12. Bordin D, Fadel CB, Moimaz SAS, Garbim CAS, Saliba NA. Estudo comparativo da satisfação de usuários e profissionais da saúde com o serviço público odontológico. Cien Saude Colet 2017; 22 (1):151-160.

13. Silva AMM, Mambrini JVM, Peixoto SV, Malta DC, Costa MFL. Uso de serviços de saúde por idosos brasileiros com e sem limitação funcional. Rev Saude Publica 2017; 51(1):1-10.

14. Araujo LUA, Gama ZAS, Nascimento SLA, Oliveira HFV, Azevedo WM, Almeida Júnior HJB. Avaliação da qualidade da atenção primária à saúde sob a perspectiva do idoso. Cien Saude Colet 2014; 19(8):35213532 .
15. Zhang X, Yu B, He T, Wang P. Status and determinants of health services utilization among elderly migrants in China. Glob Health Res Policy 2018; 21(3):3-8.

16. St Clair P, Gaudette É, Zhao H, Tysinger B, Seyedin R, Goldman DP. Using Self-reports or Claims to Assess Disease Prevalence: It's Complicated Med Care 2017; 55(8):782-788.

17. Vasconcellos MM, Gribel EB, Moraes IHS. Registros em saúde: avaliação da qualidade do prontuário do paciente na atenção básica, Rio de Janeiro, Brasil. Cad Saude Publica 2008; 24(1):173-182.

18. Roehrs A, Costa CA, Righi RDR, Oliveira KSF. Personal Health Records: A Systematic Literature Review. J Med Internet Res 2017; 13(1):1-21.

19. Jones DA, Shipman JP, Plaut DA, Selden CR. Characteristics of personal health records: findings of the Medical Library Association/National Library of Medicine Joint Electronic Personal Health Record T ask Force. J Med Libr Assoc 2010; 98(3):243-249.

20. Moraes DR, Souza JAS, Scudelari RAS, Siqueira SA, Bezerra AF, Gervázio SMD. Avaliação dos prontuários dos pacientes de uma instituição pública do município de Paraibuna do estado de São Paulo. Vita et Sanitas 2012; 1(6):2-16.

21. Brasil AMFE, Medeiros CRG, Saldanha OMFL. Estratégia saúde da família: análise dos Registros em prontuários. Cad. pedagóg 2015; 12(1):265-276.

22. Señor IC, Fernández-Alemán JL, Toval A. Are Personal Health Records Safe? A Review of Free Web-Accessible Personal Health Record Privacy Policies. J Med Internet Res 2012; 14(4):e114.

23. Bertolucci PHF, Brucki SMD, Campacci SR, Juliano Y O mini-exame do estado mental em uma população geral: impacto da escolaridade. Arq. Neuro-psiquiatr. 1994; 52(1):1-7.

24. Landis JR, Koch GG. The measurement of observer agreement for categorical data. Biometrics 1977; 33(1):159-174.

25. Morais CGX, Batista EMS, Castro JFL, Assunção SS, Castro GMO. Registros de enfermagem em prontuário e suas implicações na qualidade assistencial segundo os padrões de acreditação hospitalar: um novo olhar da auditoria. Revista ACRED 2015; 5(9):64-84.

26. Williams H, Spencer K, Sanders C, Lund D, Whitley EA, Kaye J, Dixon WG. Dynamic Consent: A Possible Solution to Improve Patient Confidence and Trust in How Electronic Patient Records Are Used in Medical Research. JMIR Med Inform 2015; 3(1):e1-7.

27. Dolan CM, Farmer LJ. Let the Record Speak: The Power of the Medical Record. J Nurse Pract 2016; 12(2):88-94.

28. Costa SM, Ferreira A, Xavier LR, Guerra PNS, Rodrigues CAQ. Referência e contrarreferência na saúde da família: percepção dos profissionais de saúde. Rev. APS 2013; 16(3):287-293.

29. MacLeod S, Musich S, Gulyas S, Cheng Y, Tkatch R, Cempellin D, Bhattarai GR, Hawkins K, Yeh CS. The impact of inadequate health literacy on patient satisfaction, healthcare utilization, and expenditures among older adults. Geriatric Nursing 2017; 38(4):334-341. 
30. Souza-Junior PRB, Freitas MPS, Antonassi GA, Szwarcwald CL. Desenho da amostra da Pesquisa Nacional de Saúde 2013. Epidemiol Serv Saúde 2015; 24(2):207-216.

31. Vieira EWR, Lima TMN, Gazzinelli A. Tempo de espera por consulta médica especializada em um município de pequeno porte de Minas Gerais, Brasil. Rev Min Enferm 2015; 19(1):65-71.

32. Soors W, Paepe P, Unger J. Management commitments and primary care: another lesson from costa rica for the world? Int J Health Serv 2014; 44(2):337-353.

33. Silva PSC, Boing AF, Peres KG. Redução das desigualdades no uso de consultas médicas no Brasil: análise das regiões Nordeste e Sudeste entre 2003 e 2008. Rev Bras Epidemiol 2015; 18(1):248-261.

34. Barbosa DCM, Mattos ATR, Corrêa MH, Faria M, Ribeiro LC, Santos LL, Ferreira JBB, Forster AC. Visita domiciliar sob a percepção dos usuários da estratégia saúde da família. Medicina (Ribeirão Preto) 2016; 49(4):360-366.

35. Riquinho DL, Pellini TV, Ramos DT, Silveira MR, Santos VCF. O cotidiano de trabalho do Agente Comunitário de Saúde: entre a dificuldade e a potência. Trab. Educ. Saúde 2018; 16(1):163-182.

36. Samudio JLP, Brant LC, Martins ACFDC, Vieira MA, Sampaio CA. Agentes Comunitários de Saúde na Atenção Primária no Brasil: multiplicidade de atividades e fragilização da formação. Trab. Educ. Saúde 2017; 15(3):745-770.

Artigo apresentado em 17/04/2018

Aprovado em 26/09/2018

Versão final apresentada em 28/09/2018 